\title{
In-depth Proteomic mapping of mouse (Mus musculus) epididymal constructive basis for sperm maturation
}

\author{
Xin Liu', Fu-Jun Liü ${ }^{1 *}$, Shao-Hua Jin², Xiao-Fang Shen ${ }^{1}$ and Yan-Wei Wang ${ }^{1}$
}

\begin{abstract}
Background: The mouse epididymis performs an essential role in sperm maturation, but global protein expression data in mouse epididymis are still lacking. Here, we reported the first in-depth gel-based profiling of mouse epididymis proteome and established a 2-DE map.

Results: A total of 832 protein spots were detected in the reproducible gels, and 625 spots corresponding to 355 unique protein entries have been successfully identified by MALDI-TOF-MS. The confidence of proteome data was validated by Western blot. Functional annotations showed that these proteins were mainly related to general metabolism, antioxidant and structural molecule activity. Immunohistochemistry disclosed two structural proteins (myosin regulatory light polypeptide 9 and alpha-2 type I collagen) continuously expressed in the myoid cell since postpartum.

Conclusion: This study provides a first-draft reference map of the mouse epididymis proteome, which will greatly expand the knowledge of the epididymal structural basis and contribute to the better understanding of those proteins in the process of mouse epididymal sperm maturation.
\end{abstract}

Keywords: Sperm maturation, Structural proteins, Mouse epididymis, Bioinformatics, Proteomics

\section{Introduction}

The epididymis is a tightly elongated tube, which connects the testis with the vas deferens. Spermatozoa produced by the testis are not yet motile or fertile. During the epididymal transit, those immature spermatozoa interact with the epididymal molecules, especially with the epididymal proteins [1]. Then the sperm surfaces undergo complex modifications, such as the loss or increase of the protein composition, and the glycosylation or phosphorylation modifications [2, 3]. Finally, these immature spermatozoa acquire the maturational capacity to survive in the female tract, move to the oviduct, penetrate the egg, and fuse with the oocyte [4]. Therefore, the epididymis is the vital organ for sperm maturation. Several important epididymal proteins have been described, such as clusterin [5], Bin1b [6], CD52 [7], ADAM7 [8], but more proteins were still expected to be

\footnotetext{
* Correspondence: cnproteome@126.com

${ }^{1}$ Central Laboratory, Yantai Yu Huang Ding Hospital/Qingdao University,

Yantai 264000, Shandong, People's Republic of China

Full list of author information is available at the end of the article
}

identified for understanding the epididymal roles in spermatozoa maturation.

Epididymal proteomes of several species have been performed such as boar [9], stallion [10], ram [11], bovine [12] and human [13]. The results identified few common epididymal proteins across different species. Therefore, the species-specific epididymal proteins exist and cannot be ignored. In this paper, we presented an in-depth two-dimensional epididymal proteome map of the mouse Mus musculus. The result would provide important information for understanding the mouse epididymal functions.

\section{Results and discussion}

Eight hundred and thirty two spots were consistently present on the two-dimensional gel (Additional file 1: Figure S1). Fig. 1 and Additional file 2: Figure S2 showed the representative two-dimensional gel0 and an annotated gel respectively. All the 832 spots were excised, digested, and analyzed by mass spectrometry. Six hundred and twenty-five spots (75.1\%) out of 832 spots were confidentially identified, and among them, 119 unique proteins 


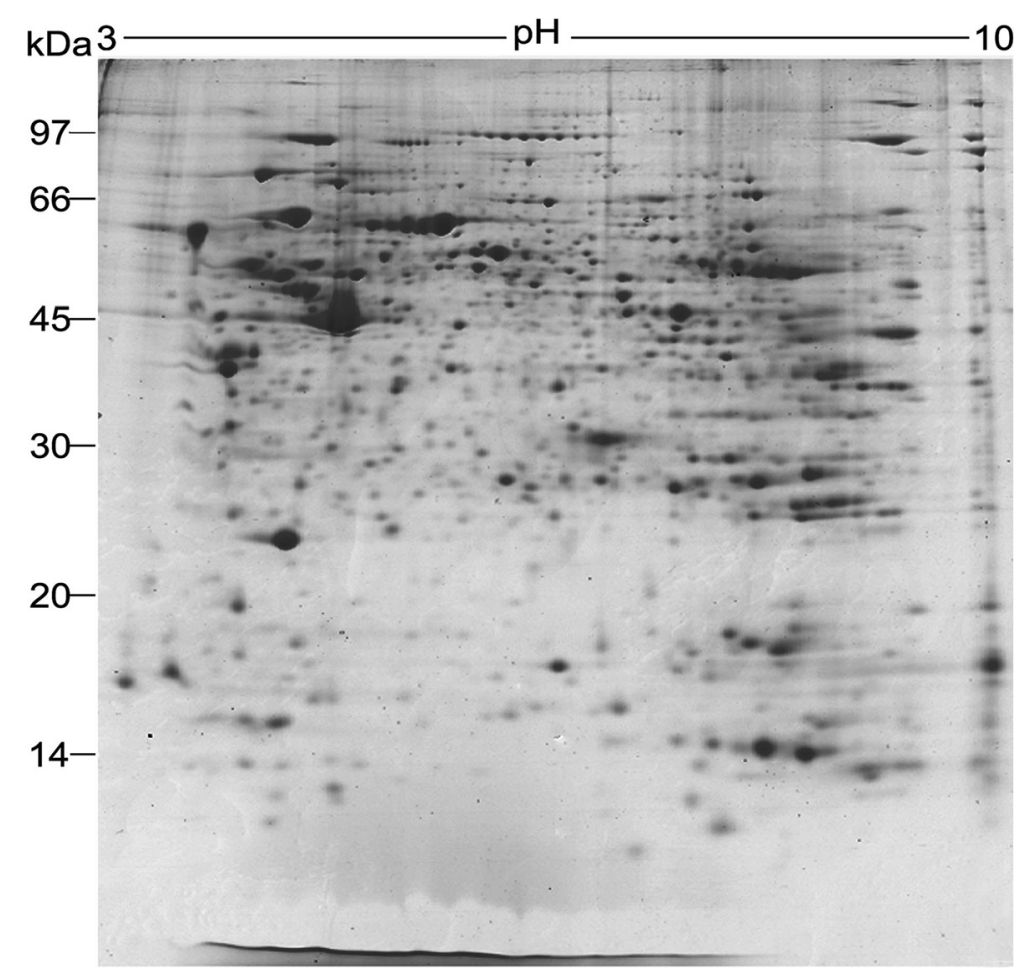

Fig. 1 A representative 2D gel of the mouse epididymal proteins

(33.5\%) were identified from more than one spot in the gel (Additional file 3: Table S1 and Additional file 4: Figure S3), 625 spots corresponded to 355 unique proteins. The molecular masses were from 8.1 to $309.2 \mathrm{kDa}$, and their isoelectric points varied between 3.86 and 11.46 .

The data confidence of mass spectrometry was validated by the analysis of western blot, and the results were in accordance with the proteomic data (Additional file 5: Figure S4). All the identified proteins were categorized by the Gene Ontology (GO) annotations, which included three aspects: molecular function, cellular component, and biological process.

Annotations for molecular function, 313 (90.7 \%) mouse epididymal proteins were assigned to 89 different functions, and then they were clustered into $13 \mathrm{GO}$ slim categories (Fig. 2a). The largest part was protein binding (19\%), followed by structural molecule activity (15\%), oxidoreductase activity (14\%), antioxidant activity (9\%), hydrolase activity (9\%), nucleotide binding (8\%), transferase activity $(7 \%)$, catalytic activity $(6 \%)$, enzyme regulator activity (4\%), and ion binding (4\%). The GO slims isomerase activity $(2 \%)$, and lyase activity $(0.8 \%)$ were the smaller part. Those proteins (15\%) with structural molecule activity might be involved in the formation of the tight junction of blood-epididymis barriers, which regulated the exchange of molecules between the epididymal lumen and the blood circulation, and created a specific epididymal microenvironment for sperm maturation
$[14,15]$. Additionally, almost $10 \%$ proteins were related to antioxidant activity, which removed excessive reactive oxygen species to provide a non-oxidative environment and protect epididymal cell DNA from oxidative damage $[16,17]$. Molecular function showed the structural-related proteins were a main part in the whole identified proteins. Immunohistochemistry analysis (Fig. 3) also indicated the structural proteins (myosin regulatory light polypeptide 9 and alpha-2 type I collagen) indeed existed at different development stages since postpartum, which were around the epididymal tubule $[18,19]$. Those structure proteins might provide the structural basis for the maintenance of epididymal sperm maturation environment, the epididymal contraction and the transport of epididymal sperm [18].

Annotations for the cellular compartments were founded for $266(77.1 \%)$ mouse epididymal proteins. Those proteins were allocated to 75 cellular compartments and were classified into $10 \mathrm{GO}$ slim categories (Fig. 2b). Most terms were assigned to cytoplasm (61\%). Cytoskeleton, vesicle, extracellular region, nucleus, membrane, extracellular matrix, and proteasome for $9 \%, 4 \%, 4 \%, 3 \%, 2 \%$, and $28 \%$ GO terms, respectively. The smallest fraction was localized to endoplasmic reticulum (1\%). The most fraction of cytoplasm (61\%) demonstrated the methodology bias of two-dimensional gel, which was inclined to the identification of cytoplasmic and soluble proteins [20].

For biological process, 285 proteins $(82.6 \%)$ were annotated by 228 cellular processes, and then were divided 


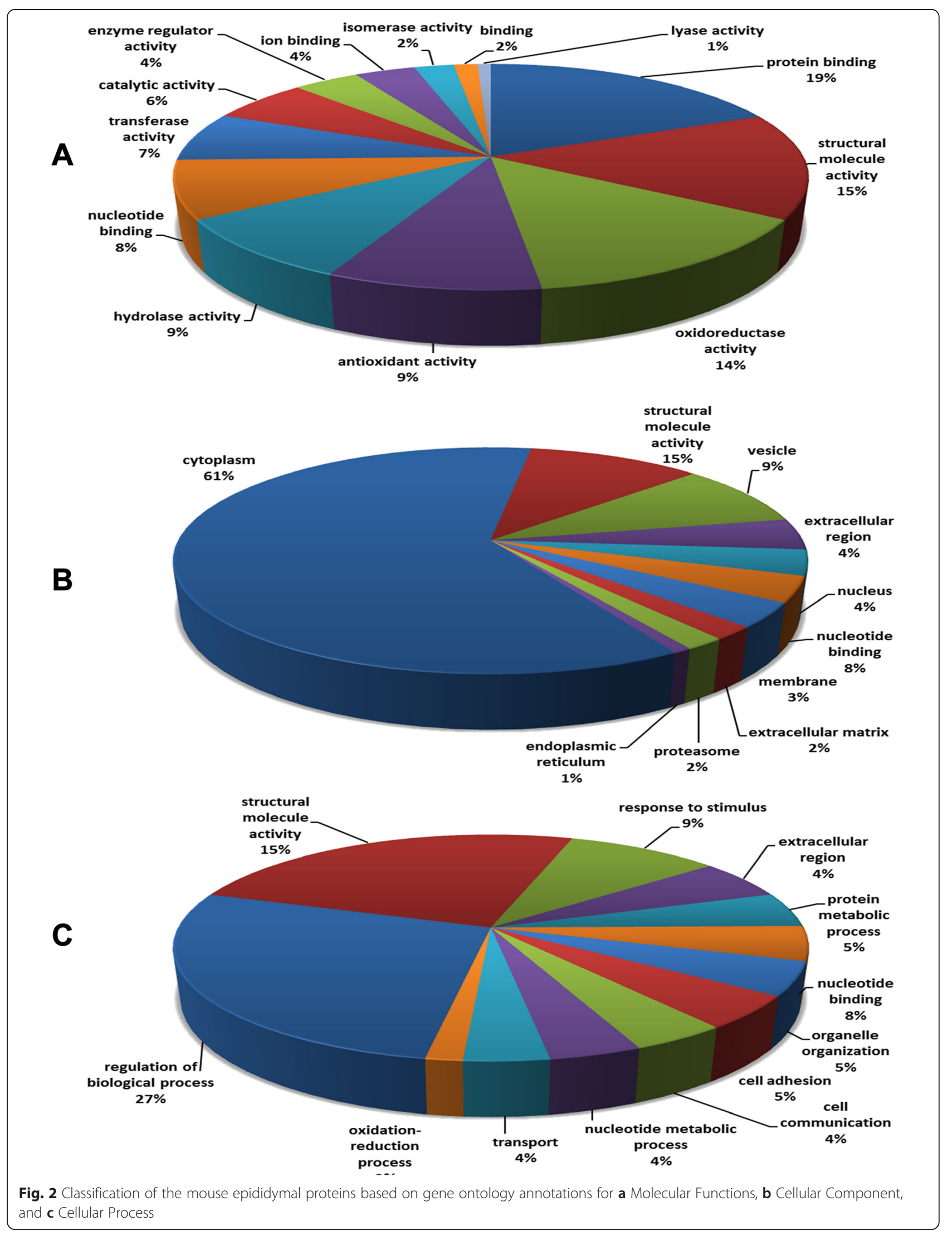




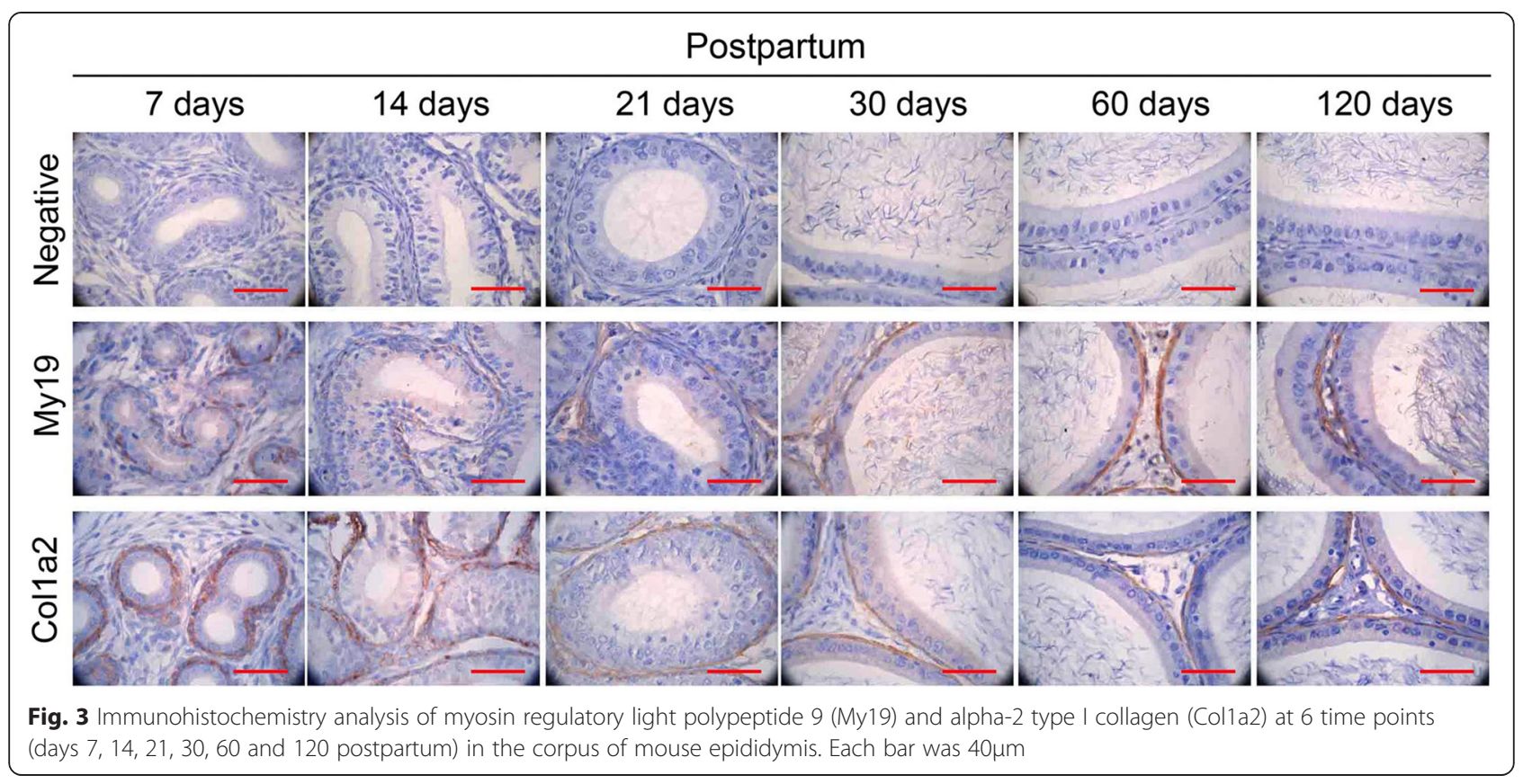

into $12 \mathrm{GO}$ slim categories (Fig. 2c). The major biological process term was regulation of biological process $(27 \%)$. The remaining terms were designed to protein metabolic process (5\%), cell adhesion (5\%), organelle organization (5\%), and transport (4\%). A small fraction was belonged to the oxidation-reduction process ( $2 \%)$. More proteins related to regulation of biological process might meet the requirements of active absorption and secretion functions of epididymal epithelium $[2,21]$.

\section{Conclusions}

This work provided the first two-dimensional map of the mouse epididymal proteome, greatly expanded the knowledge of the structural basis of the mouse epididymal sperm maturation, and facilitate biological interpretation of epididymal function in a network context. Our interest focused on proteins of the mouse epididymis because they are necessary for sperm maturation and may become potential post-testicular contraceptive targets.

\section{Materials and methods}

\section{Animal and protein extraction}

Ten adult Kunming mice (Mus musculus) were used, and all the experiments were approved by the Institutional Animal Care and Use Committee of Yantai Yu-Huang-Ding Hospital. Under anesthesia with diethyl ether, the mice were euthanized. The mice epididymides were immediately separated without blood vessels and connective tissues. Then, the mice epididymal tubules were minced with gentle pressure in the phosphate saline buffer. After agitation at $4{ }^{\circ} \mathrm{C}$ for $15 \mathrm{~min}$, the tubules were collected by centrifugation at $130 \times \mathrm{g}$ for $10 \mathrm{~min}$ at $4{ }^{\circ} \mathrm{C}$. The supernatant was removed and the tubule fragments were washed at $36 \times \mathrm{g}$ for $10 \mathrm{~min}$ at $4{ }^{\circ} \mathrm{C}$ for several times with phosphate saline buffer until no spermatozoa were found in the microscopic examination of the pellet medium. Sperm-free pellets were powdered in liquid nitrogen and dissolved in the protein lysis solution containing 7 $\mathrm{M}$ urea, $2 \mathrm{M}$ thiourea, $4 \%$ CHAPS, $65 \mathrm{mM}$ DTT at $4{ }^{\circ} \mathrm{C}$ for $2 \mathrm{~h}$. After centrifugation at $40,000 \times \mathrm{g}$ for $1 \mathrm{~h}$ at $4{ }^{\circ} \mathrm{C}$, the supernatant were transferred into a new fresh tube. Four volumes of ice-cold acetone were added into the tube and stored at $-20^{\circ} \mathrm{C}$ for $1 \mathrm{~h}$. After centrifugation at $20,000 \times \mathrm{g}$ for $1 \mathrm{~h}$ at $4{ }^{\circ} \mathrm{C}$, precipitates were washed with ice-cold acetone, air-dried, dissolved in the protein lysis buffer and stored at $-80{ }^{\circ} \mathrm{C}$ until use. Bradford assay was used to determine the protein concentration [22].

\section{2-DE and protein identification}

Eighteen cm nonlinear pH 3-10 IPG strips were used for one-dimensional IEF. After reduction and iodoacetamide, the strips were run on $12.5 \%(\mathrm{w} / \mathrm{v})$ SDS-PAGE. The gels were stained with CBB R-350 (Amersham Biosciences, Buckinghamshire, England), and were scanned by the Z320 scanner (Founder, Beijing, China). The gel maps were analyzed with the software of Imagemaster 6.0 (GE Healthcare). The electrophoresis experiments were performed in triplicate. The gel spots were excised, destained with $25 \mathrm{mM} \mathrm{NH}_{4} \mathrm{HCO}_{3} / 50 \%(\mathrm{v} / \mathrm{v}) \mathrm{ACN}$, and digested by trypsin in $25 \mathrm{mM} \mathrm{NH}_{4} \mathrm{HCO}_{3}$ at $37^{\circ} \mathrm{C}$ for $12 \mathrm{~h}$. The resultant peptides were analyzed by a Voyager DE-STR biospectrometry work station (Applied Biosystems/MDS SCIEX, Foster City, CA). The spectra data of mass spectrometry was searched against the NCBInr database (Swissprot 
Release 55.0; 356194 sequences; 127836513 residues) for Mus musculus (house mouse) with Mascot (http:// www.matrixscience.com/, MatrixScience Ltd., UK). For peptide mass fingerprinting search, only if the protein score was more than 60 and the matched peptides was greater than or equal to 4 , the protein was confirmed as a successful identification. If one gel spot corresponded to more than one protein, only the protein with the highest score was selected.

\section{Bioninformatics}

The bioinformatics analysis was carried out as our previous report [13]. The identified proteins were retrieved against the PIR database (http://pir.georgetown.edu/ pirwww/, accessed 12 August 2013). Gene ontological annotations (GO; http://www.geneontology.org/, accessed 12 August 2013) including molecular function, biological process and cellular component were clustered. Those without annotation were described as "unclassified".

\section{Western blot}

To further validate the identification confidence of proteins by mass spectrometry, nine proteins (clusterin; glyceraldehyde-3-phosphate dehydrogenase; peroxiredoxin1 ; superoxide dismutase $[\mathrm{Mn}]$; myosin regulatory light polypeptide 9; glutathione S-transferase P1; peroxiredoxin-6; alpha-2 type I collagen; superoxide dismutase $[\mathrm{Cu}-\mathrm{Zn}]$ ) were randomly selected and detected by western blot. For Western blot, $50 \mu \mathrm{g}$ of protein was separated by $12.5 \%(\mathrm{w} / \mathrm{v})$ SDS-PAGE, and then was transferred to PVDF membranes. Subsequently, the membranes were blocked with $2 \%(\mathrm{w} / \mathrm{v})$ skimmed milk for $1 \mathrm{~h}$ and were incubated with the primary antibody at RT for $1 \mathrm{~h}$. After washes by TBST for three times, membranes were incubated with HRP-conjugated anti-IgG for $1 \mathrm{~h}$. A diaminobenzidene kit (Zhong Shan Biotechnology, Beijing, China) was used to visualize the immunoreactive complexes.

\section{Immunohistochemistry}

The mouse epididymides were fixed in Bouin's solution for $10 \mathrm{~h}$, and embedded in paraffin. For antigen retrieval, the $4 \mu \mathrm{m}$-thickness sections were processed in a microwave oven for $15 \mathrm{~min}$, and $3 \%(\mathrm{v} / \mathrm{v}) \mathrm{H}_{2} \mathrm{O}_{2}$ was used to incubate the sections for $10 \mathrm{~min}$ to eliminate the endogenous peroxidases. After the antigen blocking with $3 \%$ bovine serum albumin for $30 \mathrm{~min}$, primary antibody was added overnight at $4{ }^{\circ} \mathrm{C}$. After TBS washed the sections for several times, horseradish peroxidaseconjugated anti-rabbit IgG (ZhongShan Biotechnology) was added for $1 \mathrm{~h}$ at $37^{\circ} \mathrm{C}$. The DAB kit (ZhongShan Biotechnology) was used to reveal the binding sites. Then the sections were counterstained by hematoxylin, and mounted with bright-field microscopy (DM LB2,
Leica, Nussloch, Germany). For a negative control, preimmune rabbit IgG was instead of primary antibody.

\section{Additional files}

Additional file 1: Figure S1. The replicates of 2DE gels. (JPEG $3421 \mathrm{~kb}$ )

Additional file 2: Figure S2. An annotated 2D gel of the mouse

epididymal proteins. (JPEG $372 \mathrm{~kb}$ )

Additional file 3: Table S1. 625 identified proteins of the mouse epididymis. (DOCX $139 \mathrm{~kb}$ )

Additional file 4: Figure S3. The number of isoform or splice variants of the 355 mouse epididymal proteins. Most proteins were associated with only single spots on 2D gels. Fewer proteins had up to and over 5 spots. (JPEG $28 \mathrm{~kb}$ )

Additional file 5: Figure S4. Western blotting of proteins found in mouse epididymis. Lane 1, clusterin; Lane 2, glyceraldehyde-3-phosphate dehydrogenase; Lane 3, peroxiredoxin-1; Lane 4, superoxide dismutase [Mn]; Lane 5, myosin regulatory light polypeptide 9; Lane 6, glutathione S-transferase P; Lane 7, peroxiredoxin-6; Lane 8, alpha-2 type I collagen; Lane 9, superoxide dismutase [Cu-Zn]; Lane 10, negative control. (JPEG 59 kb)

\section{Abbreviations}

2-DE: Two-dimensional gel electrophoresis; ACN: Acetonitrile; CHAPS: 3-[(3-Cholamidopropyl) dimethylammonio]-1-propanesulfonate; DTT: Dithiothreitol; CBB: Coomassie brilliant blue; IEF: Isoelectric focusing; IPG: Immobilized pH gradient; MS: Mass spectrometry; RT: Room temperature; PVDF: Polyvinylidene fluoride; TBST: Tris-buffered saline Tween-20.

\section{Competing interests}

The authors declare that they have no competing interests.

\section{Authors' contributions}

Xin Liu and Fu-Jun Liu designed the research, performed the proteomic experiment, and written the manuscript; Shao-Hua Jin, Xiao-Fang Shen, and Yan-Wei Wang carried out the western blotting and IHC analysis. All authors read and approved the final manuscript.

\section{Acknowledgement}

This work was supported by grants from the National Natural Science Foundation of China (no. 81300533) and Shandong Provincial Natural Science Foundation, China (ZR2013HQ002, ZR2014HQ068). The authors have declared no conflict of interest.

\section{Author details}

${ }^{1}$ Central Laboratory, Yantai Yu Huang Ding Hospital/Qingdao University, Yantai 264000, Shandong, People's Republic of China. ${ }^{2}$ Clinical Laboratory, Yantai Yu Huang Ding Hospital/Qingdao University, Yantai 264000,

Shandong, People's Republic of China.

Received: 7 April 2015 Accepted: 20 July 2015

Published online: 30 July 2015

\section{References}

1. Cooper TG. Sperm maturation in the epididymis: a new look at an old problem. Asian J Androl. 2007:9(4):533-9.

2. Dacheux JL, Dacheux F. New insights into epididymal function in relation to sperm maturation. Reproduction. 2013;147(2):R27-42.

3. Cornwall GA. Role of posttranslational protein modifications in epididymal sperm maturation and extracellular quality control. Adv Exp Med Biol. 2014;759:159-80.

4. Yeung $\mathrm{CH}$, Cooper TG. Developmental changes in signalling transduction factors in maturing sperm during epididymal transit. Cell Mol Biol (Noisy-le-grand). 2003:49:341-9.

5. Mattmueller DR, Hinton BT. Clusterin (SGP-2) in epididymal luminal fluid and its association with epididymal spermatozoa in androgen-deprived rats. Mol Reprod Dev. 1992;32(1):73-80. 
6. Li P, Chan HC, He B, So SC, Chung YW, Shang Q, et al. An antimicrobial peptide gene found in the male reproductive system of rats. Science. 2001;291:1783-5.

7. Yeung CH, Pérez-Sánchez F, Schröter S, Kirchhoff C, Cooper TG. Changes of the major sperm maturation-associated epididymal protein HE5 (CD52) on human ejaculated spermatozoa during incubation. Mol Hum Reprod. 2001;7(7):617-24.

8. Cornwall GA, Hsia N. ADAM7, a member of the ADAM (a disintegrin and metalloprotease) gene family is specifically expressed in the mouse anterior pituitary and epididymis. Endocrinology. 1997;138(10):4262-72.

9. Syntin P, Dacheux JL, Dacheux F. Postnatal development and regulation of proteins secreted in the boar epididymis. Biol Reprod. 1999;61:1622-35.

10. Fouchécourt S, Métayer S, Locatelli A, Dacheux F, Dacheux JL. Stallion epididymal fluid proteome: qualitative and quantitative characterization; secretion and dynamic changes of major proteins. Biol Reprod. 2000;62:1790-803.

11. Druart X, Gatti JL, Dacheux F, Dacheux UL. Analysis by two-dimensiona gel electrophoresis of ram epididymal secreted proteins. Cell Mol Biol (Noisy-le-grand). 1994:40:91-3.

12. Belleannée C, Labas V, Teixeira-Gomes AP, Gatti UL, Dacheux F, Dacheux JL. Identification of luminal and secreted proteins in bull epididymis. J Proteomics. 2011:74:59-78.

13. Li J, Liu F, Wang H, Liu X, Liu J, Li N, et al. Systematic mapping and functional analysis of a family of human epididymal secretory sperm-located proteins. Mol Cell Proteomics. 2010;9:2517-28.

14. Mital P, Hinton BT, Dufour JM. The blood-testis and blood-epididymis barriers are more than just their tight junctions. Biol Reprod. 2011;84:851-8.

15. Dubé $E_{1}$ Cyr DG. The blood-epididymis barrier and human male fertility. Adv Exp Med Bio. 2012;763:218-36.

16. Vernet P, Aitken RJ, Drevet JR. Antioxidant strategies in the epididymis. Mol Cell Endocrinol. 2004;216:31-9.

17. Yeung $\mathrm{CH}$, Wang $\mathrm{K}$, Cooper TG. Why are epididymal tumours so rare? Asian J Androl. 2012;14:465-75

18. Pelliccione F, Cordeschi G, Bocchio M, Mancini M, Sagone P, Francavilla F, et al. Immunophenotypical characterization of contractile cells in caput epididymidis of men affected by congenital or post-inflammatory obstructive azoospermia. Mol Hum Reprod. 2005:11(4):289-94.

19. Yamazaki K, Fukata H, Adachi T, Tainaka H, Kohda M, Yamazaki M, et al. Association of increased type I collagen expression and relative stromal overgrowth in mouse epididymis neonatally exposed to diethylstilbestrol. Mol Reprod Dev. 2005;72(3):291-8.

20. Pechanova O, Pechan T, Rodriguez JM, Williams WP, Brown AE. A two-dimensional proteome map of the aflatoxigenic fungus Aspergillus flavus. Proteomics. 2013;13:1513-8.

21. Cornwall GA. New insights into epididymal biology and function. Hum Reprod Update. 2009;15:213-27.

22. Hammond JB, Kruger NJ. The bradford method for protein quantitation. Methods Mol Biol. 1988:3:25-32

\section{Submit your next manuscript to BioMed Central and take full advantage of:}

- Convenient online submission

- Thorough peer review

- No space constraints or color figure charges

- Immediate publication on acceptance

- Inclusion in PubMed, CAS, Scopus and Google Scholar

- Research which is freely available for redistribution 